\title{
An improved synthesis of arenedicarboximides by phosphine-assisted annulation of arene-1,2-dicarbaldehyde with $\mathbf{N}$-substituted maleimide
}

\author{
Mitsunori Oda ${ }^{1, ~ *, ~ H a r u k i ~ S h i m o s a s a ~}{ }^{1}$, Yoshimitsu Kumai ${ }^{1}$, Akira Ohta $^{1}$, Ryuta Miyatake ${ }^{2, *}$ \\ ${ }^{1}$ Department of Chemistry, Faculty of Science, Shinshu University, Asahi 3-1-1, Matsumoto, Nagano 390-8621, Japan \\ ${ }^{2}$ Centre for Environmental Conservation and Research Safety, University of Toyama, Gofuku 3190, Toyama 930-8555, Japan
}

Email address:

mituoda@shinshu-u.ac.jp (M. Oda), miyatake@ctg.u-toyama.ac.jp (R. Miyatake)

To cite this article:

Mitsunori Oda, Haruki Shimosasa, Yoshimitsu Kumai, Akira Ohta, Ryuta Miyatake. An Improved Synthesis of Arenedicarboximides by Phosphine-Assisted Annulation of Arene-1,2-Dicarbaldehyde with $N$-Substituted Maleimide. Modern Chemistry.

Vol. 2, No. 4, 2014, pp. 29-35. doi: 10.11648/j.mc.20140204.11

\begin{abstract}
Various arene-1,2-dicarbaldehydes react with $N$-substituted maleimides in the presence of trialkylphosphine in refluxing dioxane to afford the title compounds in good yields, which are better than those in a similar reaction using triethylphosphite reported by Haddadin et al. Many products were isolated by direct filtration from the reaction mixtures and washing with a suitable solvent. Application of the method to synthesis of diimides is also reported.
\end{abstract}

Keywords: Maleimides, Arene-1,2-Dicarbaldehydes, Morita-Baylis-Hillman Reaction, Intramolecular Wittig Reaction

\section{Introduction}

Arenedicarboximides show interesting photochemical behaviors, such as photoreduction [1,2] and photocycloaddition [3,4], and also unique photophysical properties [5-8]. Recently, Heagy et al. reported that dual fluorescent 2,3-naphthalenedicaboximides could be used in ratiometric DNA detection and as white organic light-emitting devices (OLEDs) [9], and syntheses of various di- and polydicarboximides for OLEDs and organic field-effect transistors have been continuously reported [10-12], indicating that arenedicarboximides are promising agents for biochemical reactions and electronic materials. $N$-Substituted arenedicarboximides can be obtained by various simple ways, such as condensation of arenedicarboxylic anhydride with amine [11,13-15] and Gabriel reaction of $N$-unsubstituted arenedicarboximide with alkyl or aryl halide [16-18]. When these anhydride and $\mathrm{N}$-unsubstituted dicarboximide are commercially available or easily accessible, these methods are superior. Moreover, an approach by Diels-Alder reaction via arene-o-quinodimethane or isobenzofuran constructing concomitantly another benzene ring has been reliable [19-22]. Besides, Haddadin et al. reported an interesting synthetic reaction, in which $o$-phthaldehyde (1) condenses with $N$-phenylmaleimide (3) in the presence of triethylphosphite to give $N$-phenyl-2,3-naphthalenedicarboximide (2) in a satisfactory yield (62\%) (Scheme 1) [23]. However, they also reported that the reaction of 2,3-naphthaldehyde under similar reaction conditions resulted in a low yield $(35 \%)$ of the anthracenedicarboximide. Notwithstanding<smiles>O=Cc1ccccc1C=O</smiles>

1

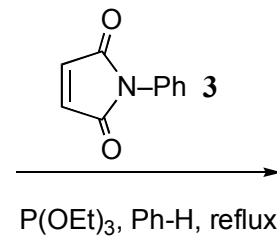

Scheme 1. A synthetic method of 2 by Haddadin et al. the fascinating methodology of Haddadin et al., there has been no work of its improvement reported for a long time. Herein we describe results of our effort to improve this reaction with various phosphorus reagents and solvents.

\section{Results and Discussion}

First, the reaction of 1 and 3 with various solvents and phosphorus reagents under refluxing conditions was examined. 
The results are presented in Table 1. It should be noted that the yields in the Table are ones isolated directly from reaction mixture just by filtration and washing with cold ether. Among solvents used, the reaction in dioxane gave a better yield than those in other solvents (Entry 1-5). Triphenylphosphine assisted the reaction as much as triethylphosphite (Entry 5), and bulky tri-tert-butyl and tri-o-tolylphosphines were entirely ineffective (Entry 6-7). The satisfactory yields were observed when tri-n-butyl-, tricyclohexyl-, and tri- $n$-octylphosphines were used (Entry 8-10). Under the conditions with a slight excess of tri- $n$-octylphosphine and 3 , the product 2 was obtained in the best yield of $89 \%$ (Entry 11 ), which shows a clear improvement compared with the result of Haddadin et al.

Table 1. Results of the reaction of 1 and $3^{a}$

\begin{tabular}{llll}
\hline Entry & P reagent $^{\mathbf{b}}$ & Solvent/reaction time & $\begin{array}{l}\text { Yield of 2 } \\
(\%)^{\mathbf{c}}\end{array}$ \\
\hline 1 & $\mathrm{P}(\mathrm{OEt})_{3}$ & benzene/ $15 \mathrm{~min}$ & $62^{\mathrm{d}}$ \\
2 & $\mathrm{P}(\mathrm{OEt})_{3}$ & $\mathrm{CH}_{3} \mathrm{CN} / 6 \mathrm{~h}$ & 22 \\
3 & $\mathrm{P}(\mathrm{OEt})_{3}$ & $\mathrm{EtOH} / 4 \mathrm{~h}$ & 23 \\
4 & $\mathrm{P}(\mathrm{OEt})_{3}$ & dioxane/ $1.5 \mathrm{~h}$ & 75 \\
5 & $\mathrm{PPh}$ & dioxane/ $2 \mathrm{~h}$ & 74 \\
6 & $\mathrm{P}(o \text {-tol })_{3}$ & dioxane/ $2 \mathrm{~h}$ & 0 \\
7 & $\mathrm{P}(t \text {-butyl })_{3}{ }^{\mathrm{e}}$ & dioxane/ $2 \mathrm{~h}$ & 0 \\
8 & $\mathrm{P}(c \text {-hexyl })_{3}$ & dioxane/ $2 \mathrm{~h}$ & 79 \\
9 & $\mathrm{P}(n \text {-butyl })_{3}$ & dioxane/ $2 \mathrm{~h}$ & 79 \\
10 & $\mathrm{P}(n \text {-octyl })_{3}$ & dioxane/ $0.5 \mathrm{~h}$ & 83 \\
11 & $\mathrm{P}(n \text {-octyl })_{3}$ & dioxane/ $0.5 \mathrm{~h}$ & $89^{\mathrm{f}}$ \\
12 & $\mathrm{P}(n \text {-octyl })_{3}$ & dioxane/ $0.5 \mathrm{~h}$ & $88^{\mathrm{g}}$ \\
\hline
\end{tabular}

a) An equivalent of 3 to 1 was used, b) 1.2 equivalents of phosphorus reagent to 1 were used, c) isolated yield directly from reaction mixture, d) taken from ref. [22], e) $\mathrm{P}(t \text {-butyl })_{3} \mathrm{HBF}_{4}$ salt was used in the presence of an equivalent of DBU, f) 1.1 equivalents of 3 to 1 were used, g) 1.2 equivalents of 3 and 1.3 equivalents of phosphine reagent to 1 were used

Secondly, various $N$-substituted maleimides were subjected to the reactions with 1 , 4,5-dimethyl-o-phthaldehyde (4) [24] and 4,5-dichloro- $o$-phthaldehyde (5) [24,25] under the conditions of entry 11 in Table 1 . Structures of the substituted 2,3-naphthalenedicarboximides synthesized are shown in Figure 1. The sixteen imide products were obtained in a range of $71-89 \%$ yields, indicating effectiveness of the improved procedure for a variety of naphthalenedicaboximides. The products were isolated mainly by filtration from the reaction mixture. However, some products soluble in dioxane to some extent were obtained partly by chromatography purification of the filtrate. We also applied our procedure to synthesis of 2,3-anthracenedicarboximides. The results are shown in Scheme 2. From 2,3-naphthaldehyde (22), the dicarboximides (23-27) were synthesized in good yields (Scheme 2). Particularly, the yield of 23 is more than twice of that

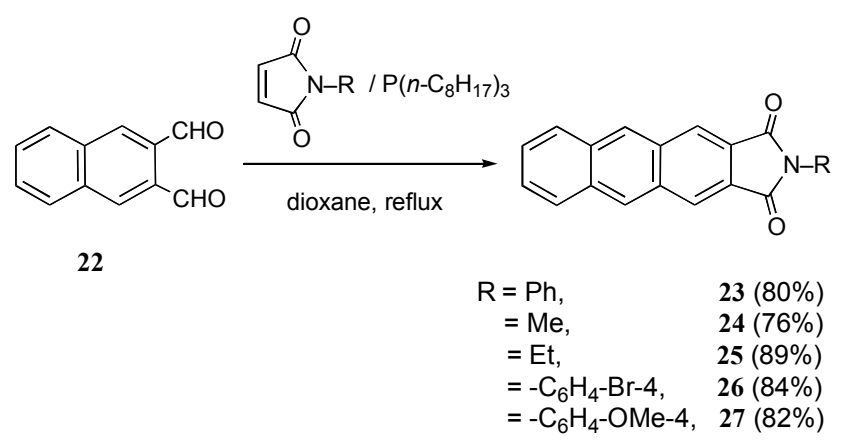

Scheme 2. Synthesis of $N$-substituted 2,3-anthracenedicarboximides.

reported by Haddadin et al. Furthermore, our protocol developed herein was applied to synthesis of diimides. Under the same conditions compounds 28 and 29 [26] react with 1 to give diimides 31 and 32 in good yields, respectively, and 30 reacts with 4 to give 33 in a moderate yield (Scheme 3 ).

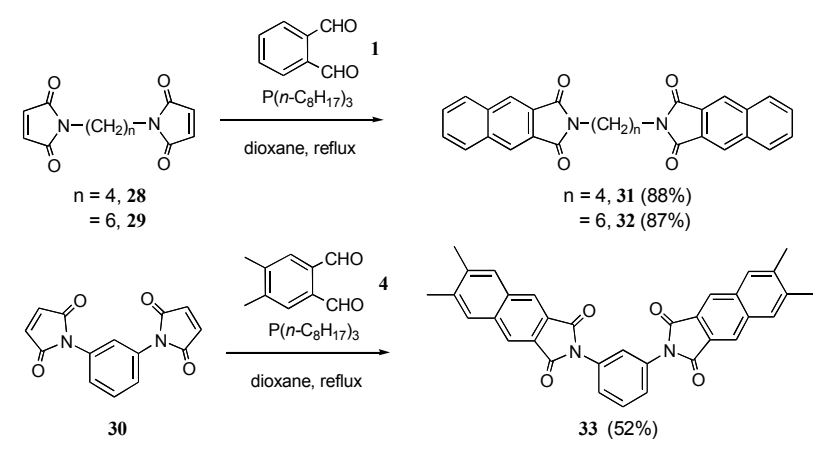

Scheme 3. Application to synthesis of diimide compounds 31-33.
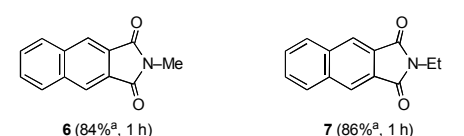

9(81\%,1h)

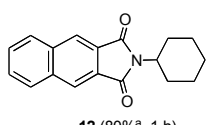

$12(80 \%, 1 \mathrm{~h})$
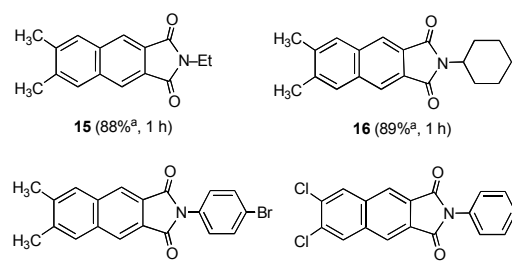

$18(79 \%$ a 1 h)
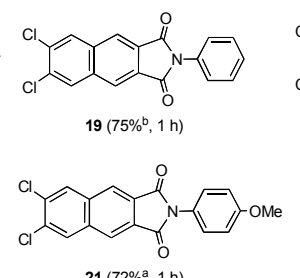
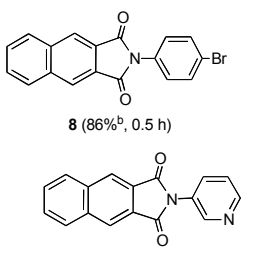

$11(82 \%$ a, $1 \mathrm{~h})$
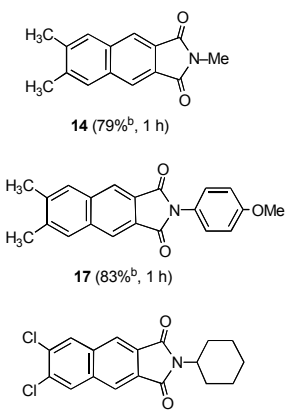

$20\left(71 \%,{ }^{a}, 1 \mathrm{~h}\right)$ $21\left(72 \%,{ }^{a}, 1 \mathrm{~h}\right)$

Figure 1. Various dicarboximides obtained under reaction conditions of entry 12 in Table 1 with the corresponding dicarbaldehyde and $N$-substituted maleimides. Yield and reaction time are shown in parentheses. ${ }^{a)}$ Isolated yield mainly by filtration from reaction mixture and partly by chromatography purification of the filtrate, ${ }^{b)}$ isolated yield directly by filtration from reaction mixture and washing with cold ether. 
In Scheme 4, the possible reaction routes to 2 from 1 and $N$-substrtuted maleimide in the presence of phosphine are shown. As previously suggested by Haddadin et al., the reaction may proceed via the Morita-Baylis-Hillman reaction [27] to produce 36 , followed by the intramolecular Wittig condensation (route A). Besides, route $\mathrm{B}$ involving intermolecular Wittig reaction of 1 with 38 , being able to be formed from maleimide and phosphine, and subsequent intramolecular aldol condensation of 39 is possible. Since the reaction of the preformed Wittig reagent 38 [28] with 1 in refluxing dioxane was found to be very slow compared with the results in Table 1 . Only a small amount of $2(12 \%$ in a case with the tri- $n$-octylphosphorane reagent) was observed even after prolonged reaction times. Therefore, a process through the route $\mathrm{B}$ must be negligible at least under the conditions. Further mechanistic studies to obtain decisive evidences for the reaction mechanism are now under progress.

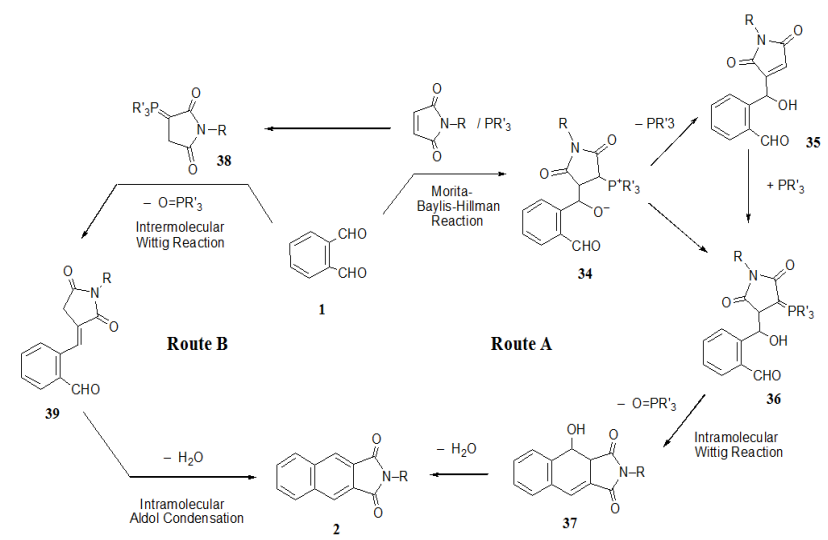

Scheme 4. Possible reaction routes to 2 from 1 and $N$-substituted maleimide in the presence of phosphine.

\section{Experimental}

\subsection{General Remarks}

Melting points were measured on a Yanaco MP-3 and are uncorrected. IR spectra were recorded on JEOL Diamond-20 and JASCO FT/IR-4100 spectrometers. UV spectra were measured on a Shimadzu UV-2550 spectrometer. ${ }^{1} \mathrm{H}-$ and ${ }^{13} \mathrm{C}$-NMR spectra were recorded on JEOL $\lambda 400$ and ECA500 spectrometers. Chemical shift values of tetramethylsilane ( $\delta=$ $0 \mathrm{ppm})$ for ${ }^{1} \mathrm{H}-\mathrm{NMR}$ spectra and $\mathrm{CDCl}_{3}(\delta=77.0 \mathrm{ppm})$ for ${ }^{13} \mathrm{C}$-NMR spectra were used as internal standard. Mass spectra were measured on a JMS-700 mass spectrometer. Column chromatography was performed with Silica gel $60 \mathrm{~N}$ from Kanto Chem. Dioxane was purchased from Kanto Chem. and was distilled over $\mathrm{CaH}_{2}$. o-Phthalaldehyde, triethylphophite, triphenylphosphine, tricyclohexylphosphine, $\mathrm{N}$-phenylmaleimide, $\mathrm{N}$-methylmaleimide, $\mathrm{N}$-ethylmaleimide, $N$-cyclohexylmaleimide, and $N, N$ '-(m-phenylene)dimale-imide were purchased from Tokyo Chemical Industry, Inc. N-(3-Pyridyl)maleimide, $N$-(4-bromophenyl)maleimide,

$\mathrm{N}$-(4-methoxyphenyl)maleimide were prepared according to a procedure reported by Cava et al. [29] N,N'-Tetramethyleneand -hexamethylenedimaleimides are prepared by the method of Tona et al. [26] 2,3-Naphthaldehyde was prepared from phthalaldehyde and 2,5-dimethoxytetrahydrofuran by a method of Lepage et al. [30] 4,5-Dimethyl-o-phthalaldehyde (4) was prepared according to the method of Farooq. [24] 4,5-Dichloro-o-phthalaldehyde (5) was prepared by the method of Chen et al. [25]

\subsection{General Procedure}

To solution of dicarbaldehyde $(0.5 \mathrm{mmol})$ and $\mathrm{N}$-substituted maleimide $(0.55 \mathrm{mmol})$ in $2 \mathrm{ml}$ of dry dioxane was added tri- $n$-octylphosphine $(0.60 \mathrm{mmol})$. The reaction mixture was refluxed on a preheated oil bath under nitrogen atmosphere for $0.5-3 \mathrm{~h}$, and was cooled to ice-bath temperature. The crystals formed were collected by suction filtration and washed well with cold ether to give a product. If necessary, the filtrate was concentrated under vacuum and the residue was purified by silica gel chromatography with a solvent system of chloroform/ethyl acetate (or chloroform/ethanol).

\subsection{N-Phenyl-2,3-naphthalimide (2)}

Colorless prisms, m.p. $284-285{ }^{\circ} \mathrm{C}$ (lit. [23] 279-280 ${ }^{\circ} \mathrm{C}$ ). ${ }^{1} \mathrm{H} \mathrm{NMR}\left(400 \mathrm{MHz}, \mathrm{CDCl}_{3}\right) \delta=8.41(\mathrm{~s}, 2 \mathrm{H}), 8.11(\mathrm{~m}, 2 \mathrm{H})$, $7.74(\mathrm{~m}, 2 \mathrm{H}), 7.54(\mathrm{tm}, J=7.0 \mathrm{~Hz}, 2 \mathrm{H}), 7.50(\mathrm{dm}, J=7.0 \mathrm{~Hz}$, $2 \mathrm{H}), 7.43(\mathrm{tm}, J=7.0 \mathrm{~Hz}, 1 \mathrm{H}) \mathrm{ppm} .{ }^{13} \mathrm{C}$ NMR $(100 \mathrm{MHz}$, $\left.\mathrm{CDCl}_{3}\right) \delta=167.0,135.7,131.9,130.4,129.4,129.1,128.2$, 127.5, 126.7, 125.3.ppm.

\subsection{N-Methyl-2,3-naphthalimide (6)}

Colorless microcrystals, m.p. 239-242 ${ }^{\circ} \mathrm{C}$ (lit. [4] 215-217 ${ }^{\circ} \mathrm{C}$, [31] 240-242 $\left.{ }^{\circ} \mathrm{C}\right) .{ }^{1} \mathrm{H}$ NMR $\left(400 \mathrm{MHz}, \mathrm{CDCl}_{3}\right) \delta=8.32(\mathrm{~s}$, $2 \mathrm{H}), 8.05(\mathrm{~m}, 2 \mathrm{H}), 7.69(\mathrm{~m}, 2 \mathrm{H}), 3.25(\mathrm{~s}, 3 \mathrm{H}) \mathrm{ppm} .{ }^{13} \mathrm{C} \mathrm{NMR}$ $\left(100 \mathrm{MHz}, \mathrm{CDCl}_{3}\right) \delta=168.1,135.4,130.3,129.1,127.9$, 124.6, $24.2 \mathrm{ppm}$.

\subsection{N-Ethyl-2,3-naphthalimide (7)}

Colorless microcrystals, m.p. $185-186{ }^{\circ} \mathrm{C} .{ }^{1} \mathrm{H}$ NMR (400 $\left.\mathrm{MHz}, \mathrm{CDCl}_{3}\right) \delta=8.32(\mathrm{~s}, 2 \mathrm{H}), 8.05(\mathrm{~m}, 2 \mathrm{H}), 7.69(\mathrm{~m}, 2 \mathrm{H})$, $3.82(\mathrm{q}, J=7.0 \mathrm{~Hz}, 2 \mathrm{H}), 1.32(\mathrm{q}, J=7.0 \mathrm{~Hz}, 2 \mathrm{H}) \mathrm{ppm} .{ }^{13} \mathrm{C}$ NMR $\left(100 \mathrm{MHz}, \mathrm{CDCl}_{3}\right) \delta=167.9,135.4,130.2,129.1,128.0$, 124.5, 33.1, 13.9 ppm. IR (KBr) $v=1760$ (vs), 1707 (vs) $\mathrm{cm}^{-1}$. $\mathrm{UV}(\mathrm{MeOH}) \lambda=215(\log \varepsilon=4.36), 239 \mathrm{sh}(4.38), 254$ (4.57), 256 (4.58), 259 (4.58), 281 (3.78), 290 (3.79), 332sh (3.14), 344 (3.39), $357(3.53) \mathrm{nm}$. MS $m / z(\%)=225\left(\mathrm{M}^{+}\right.$, 57), 211 (14), 210 (100), 197 (7), 183 (7), 155 (13), 126 (16), 91 (10), 63 (7). HRMS Cald for $\mathrm{C}_{14} \mathrm{H}_{11} \mathrm{NO}_{2}\left(\mathrm{M}^{+}\right)$225.0790, found 225.0789 .

\subsection{N-(4-Bromophenyl)-2,3-naphthalimide (8)}

Colorless microcrystals, m.p. $>300{ }^{\circ} \mathrm{C} .{ }^{1} \mathrm{H}$ NMR $(400 \mathrm{MHz}$, DMSO-d $\left.d_{6}\right) \delta=8.64(\mathrm{~s}, 2 \mathrm{H}), 8.32(\mathrm{~m}, 2 \mathrm{H}), 7.82(\mathrm{~m}, 2 \mathrm{H})$, $7.77(\mathrm{~d}, J=8.8 \mathrm{~Hz}, 2 \mathrm{H}), 7.49(\mathrm{~d}, J=8.8 \mathrm{~Hz}, 2 \mathrm{H}) \mathrm{ppm} .{ }^{13} \mathrm{C}$ NMR (100 MHz, DMSO- $\left.d_{6}\right) \delta=166.3,135.1,131.7,131.3$, 
$130.2,129.33,129.28,127.3,124.8,121.0 \mathrm{ppm}$. IR (KBr) $v$ $=1787(\mathrm{~s}), 1719(\mathrm{vs}) \mathrm{cm}^{-1}$. UV $(\mathrm{MeOH}) \lambda=203(\log \varepsilon=$ 4.12), 217 (4.15), 245sh (4.25), 261 (4.37), 338 (2.98), 359 (2.77) nm. MS $m / z(\%)=353\left(\mathrm{M}^{+}, 100\right), 351\left(\mathrm{M}^{+}, 100\right), 309$ (23), 307 (23), 228 (22), 227 (14), 153 (8), 136 (19), 126 (48). HRMS Cald for $\mathrm{C}_{18} \mathrm{H}_{10}{ }^{79} \mathrm{BrNO}_{2}\left(\mathrm{M}^{+}\right)$350.9895, found 350.9894 .

\subsection{N-(4-Methoxyphenyl)-2,3-naphthalimide (9) [6]}

Yellowish microcrystals, m.p. $245-246{ }^{\circ} \mathrm{C} .{ }^{1} \mathrm{H}$ NMR (400 $\left.\mathrm{MHz}, \mathrm{CDCl}_{3}\right) \delta=8.44(\mathrm{~s}, 2 \mathrm{H}), 8.09(\mathrm{~m}, 2 \mathrm{H}), 7.73(\mathrm{~m}, 2 \mathrm{H})$, $7.40(\mathrm{dm}, J=8.4 \mathrm{~Hz}, 2 \mathrm{H}), 7.05(\mathrm{dm}, J=8.4 \mathrm{~Hz}, 2 \mathrm{H}), 3.86(\mathrm{~s}$, $3 \mathrm{H}) \mathrm{ppm} .{ }^{13} \mathrm{C}$ NMR $\left(100 \mathrm{MHz}, \mathrm{CDCl}_{3}\right) \delta=167.3,159.3$, $135.6,130.3,129.3,127.9,125.1,124.5,114.5,55.5$ ppm. $\mathrm{UV}(\mathrm{MeOH}) \lambda=217(\log \varepsilon=4.51), 225 \mathrm{sh}(4.43), 260$ (4.72), 281sh (4.30), 342 (3.42), $358(3.45) \mathrm{nm}$.

\subsection{N-(4-Nitrophenyl)-2,3-naphthalimide (10)}

Colorless solids, m.p. $>300{ }^{\circ} \mathrm{C}$. ${ }^{1} \mathrm{H}$ NMR $(400 \mathrm{MHz}$, DMSO $-d_{6}$ at $\left.130{ }^{\circ} \mathrm{C}\right) \delta=8.59(\mathrm{~s}, 2 \mathrm{H}), 8.37(\mathrm{~d}, J=8.8 \mathrm{~Hz}$, $2 \mathrm{H}), 8.29(\mathrm{~m}, 2 \mathrm{H}), 7.88(\mathrm{~d}, J=8.8 \mathrm{~Hz}, 2 \mathrm{H}), 7.81(\mathrm{~m}, 2 \mathrm{H})$ ppm. ${ }^{13} \mathrm{CNMR}\left(100 \mathrm{MHz}, \mathrm{DMSO}-d_{6}\right.$ at $\left.130{ }^{\circ} \mathrm{C}\right) \delta=165.3$, 146.1, 137.6, 134.9, 129.7, 128.9, 127.0, 126.6, 124.6, 123.3 ppm. IR (KBr) $v=1771(\mathrm{~s}) ,1749(\mathrm{~s})$,1723 (vs) $\mathrm{cm}^{-1}$. MS $m / z(\%)=318\left(\mathrm{M}^{+}, 100\right), 288$ (19), 274 (25), 272 (14), 228 (25), 227 (9), 154 (9), 126 (52). HRMS calcd for $\mathrm{C}_{18} \mathrm{H}_{10} \mathrm{~N}_{2} \mathrm{O}_{4}$ $\left(\mathrm{M}^{+}\right)$318.0641, found 318.0638 .

\subsection{N-(3-Pyridyl)-2,3-naphthalimide (11)}

Colorless solids, m.p. 244-246 ${ }^{\circ} \mathrm{C} .{ }^{1} \mathrm{H}$ NMR $(500 \mathrm{MHz}$, $\left.\mathrm{CDCl}_{3}\right) \delta=8.85(\mathrm{dd}, J=2.5,0.7 \mathrm{~Hz}, 1 \mathrm{H}), 8.66(\mathrm{dd}, J=4.9$, $1.7 \mathrm{~Hz}, 1 \mathrm{H}), 8.48(\mathrm{~s}, 2 \mathrm{H}), 8.12(\mathrm{~m}, 2 \mathrm{H}), 7.90$ (ddd, $J=8.2$, 2.5, $1.7 \mathrm{~Hz}, 1 \mathrm{H}), 7.76(\mathrm{~m}, 2 \mathrm{H}), 7.48(\mathrm{ddd}, J=8.2 .4 .9,0.7 \mathrm{~Hz}$ 1H) ppm. ${ }^{13} \mathrm{CNMR}\left(126 \mathrm{MHz}, \mathrm{CDCl}_{3}\right) \delta=166.5,148.8$, 147.4, 135.7, 133.6, 130.4, 129.6, 129.0, 127.0, 125.7, 123.6 ppm. IR (KBr) $v=1784(\mathrm{~m}), 1766(\mathrm{~m}), 1715$ (vs), 1709 (vs) $\mathrm{cm}^{-1}$. UV $\left(\mathrm{CH}_{3} \mathrm{CN}\right) \lambda=261(\log \varepsilon=4.83), 294 \mathrm{sh}$ (3.99), 343 (3.42), 359 (3.57) nm. MS $m / z(\%)=274\left(\mathrm{M}^{+}, 100\right), 229$ (54), 126 (48). EA $\left(\mathrm{C}_{17} \mathrm{H}_{10} \mathrm{~N}_{2} \mathrm{O}_{2}\right)$ Calcd C; 74.44, H; 3.67, N; $10.21 \%$, Found $\mathrm{C} ; 74.03, \mathrm{H} ; 3.72, \mathrm{~N} ; 10.20 \%$.

\subsection{N-Cyclohexyl-2,3-naphthalimide (12)}

Colorless solids, m.p. 208-209 ${ }^{\circ} \mathrm{C}$. ${ }^{1} \mathrm{HNMR}(500 \mathrm{MHz}$, $\left.\mathrm{CDCl}_{3}\right) \delta=8.30(\mathrm{~s}, 2 \mathrm{H}), 8.04(\mathrm{~m}, 2 \mathrm{H}), 7.68(\mathrm{~m}, 2 \mathrm{H}), 4.20(\mathrm{tt}$, $J=12.3,3.4 \mathrm{~Hz}, 1 \mathrm{H}), 2.28(\mathrm{qd}, J=12.3,3.4 \mathrm{~Hz}, 2 \mathrm{H}), 1.77$ $(\mathrm{dm}, J=12.3 \mathrm{~Hz}, 2 \mathrm{H}), 1.71(\mathrm{dm}, J=12.3 \mathrm{~Hz}, 1 \mathrm{H}), 1.40$ (qt, $J=12.3,3.4 \mathrm{~Hz}, 2 \mathrm{H}), 1.32(\mathrm{tt}, J=12.3,3.4 \mathrm{~Hz}, 1 \mathrm{H}) \mathrm{ppm}$. ${ }^{13} \mathrm{CNMR}\left(126 \mathrm{MHz}, \mathrm{CDCl}_{3}\right) \delta=168.3,135.6,130.3,129.1$, $128.1,124.4,51.3,29.9,26.2,25.3 \mathrm{ppm}$. IR (KBr) $v=1761$ (s), $1748(\mathrm{~m}), 1700(\mathrm{vs}) \mathrm{cm}^{-1}$. MS $m / z(\%)=279\left(\mathrm{M}^{+}, 55\right)$, 236 (28), 198 (100), 180 (30), 155 (10), 127 (10). HRMS calcd for $\mathrm{C}_{18} \mathrm{H}_{17} \mathrm{NO}_{2}\left(\mathrm{M}^{+}\right)$279.1259, found 279.1261.

\subsection{6,7-Dimethyl-N-phenyl-2,3-naphthalimide (13)}

Colorless solids, m.p. $>300{ }^{\circ} \mathrm{C}$. ${ }^{1} \mathrm{HNMR}(500 \mathrm{MHz}$,
$\left.\mathrm{CDCl}_{3},\right) \delta=8.33(\mathrm{~s}, 2 \mathrm{H}), 7.83(\mathrm{~s}, 2 \mathrm{H}), 7.53(\mathrm{tm}, J=7.2 \mathrm{~Hz}$, $2 \mathrm{H}), 7.49(\mathrm{dm}, J=7.2 \mathrm{~Hz}, 2 \mathrm{H}), 7.41(\mathrm{tm}, J=7.2 \mathrm{~Hz}, 1 \mathrm{H})$, $2.51(\mathrm{~s}, 6 \mathrm{H}) \mathrm{ppm} .{ }^{13} \mathrm{CNMR}\left(126 \mathrm{MHz}, \mathrm{CDCl}_{3}\right.$ at $\left.50{ }^{\circ} \mathrm{C}\right) \delta=$ $167.4,140.0,134.8,132.4,130.1,129.2,128.2,127.1,126.8$, 124.4, 20.5 ppm. UV $(\mathrm{MeOH}) \lambda=218(\log \varepsilon=4.39)$, 257sh (4.48), 272 (4.72), 295sh (3.93), 321sh (3.44), 352 (3.32), $365(3.46) \mathrm{nm}$. IR $(\mathrm{KBr}) v=1775(\mathrm{~m}), 1763(\mathrm{~m})$, 1735 (s), $1712(\mathrm{~s}) \mathrm{cm}^{-1}$. MS $\mathrm{m} / z(\%)=301\left(\mathrm{M}^{+}, 100\right), 257$ (38), 242 (10), 154 (9). HRMS calcd for $\mathrm{C}_{20} \mathrm{H}_{15} \mathrm{NO}_{2}\left(\mathrm{M}^{+}\right)$ 301.1103 , found 301.1101 .

\subsection{6,7-Dimethyl-N-methyl-2,3-naphthalimide (14)}

Colorless solids, m.p. 246-249 ${ }^{\circ} \mathrm{C}$. ${ }^{1} \mathrm{HNMR}(500 \mathrm{MHz}$, $\left.\mathrm{CDCl}_{3}\right) \delta=8.19(\mathrm{~s}, 2 \mathrm{H}), 7.77(\mathrm{~s}, 2 \mathrm{H}), 3.23(\mathrm{~s}, 3 \mathrm{H}), 2.48(\mathrm{~s}$, $6 \mathrm{H}) \mathrm{ppm} .{ }^{13} \mathrm{CNMR}\left(126 \mathrm{MHz}, \mathrm{CDCl}_{3}\right) \delta=168.6,139.7$, $134.3,130.0,127.3,123.7,24.3,20.5 \mathrm{ppm}$. IR (KBr) $v=$ 1770 (s), 1716 (vs), 1709 (vs) cm $\mathrm{cm}^{-1}$. MS m/z (\%) = $239\left(\mathrm{M}^{+}\right.$, 100), 211 (12), 195 (45), 154 (19), 139 (10). HRMS calcd for $\mathrm{C}_{15} \mathrm{H}_{13} \mathrm{NO}_{2}\left(\mathrm{M}^{+}\right)$239.0946, found 239.0947.

\subsection{N-Ethyl-6,7-dimethyl-2,3-naphthalimide (15)}

Colorless plates, m.p. 229-230 ${ }^{\circ} \mathrm{C} .{ }^{1} \mathrm{HNMR}(500 \mathrm{MHz}$, $\mathrm{CDCl}_{3}$,) $\delta=8.20(\mathrm{~s}, 2 \mathrm{H}), 7.78(\mathrm{~s}, 2 \mathrm{H}), 3.80(\mathrm{q}, J=7.3 \mathrm{~Hz}$, 2H), $2.48(\mathrm{~s}, 6 \mathrm{H}), 1.31(\mathrm{t}, J=7.3 \mathrm{~Hz}, 3 \mathrm{H}) \mathrm{ppm} .{ }^{13} \mathrm{CNMR}$ $\left(126 \mathrm{MHz}, \mathrm{CDCl}_{3}\right) \delta=168.4,139.7,134.4,130.0,127.4$, 123.7, 33.2, 20.5, $14.1 \mathrm{ppm}$. IR (KBr) $v=1769$ (s), 1740 (s), 1717 (vs), 1708 (vs) $\mathrm{cm}^{-1}$. MS $m / z(\%)=253\left(\mathrm{M}^{+}, 64\right), 238$ (100), 225 (13), 154 (11). HRMS calcd for $\mathrm{C}_{16} \mathrm{H}_{15} \mathrm{NO}_{2}\left(\mathrm{M}^{+}\right)$ 253.1103, found 253.1103.

\subsection{N-Cyclohexyl-6,7-dimethyl-2,3-naphthalimide (16)}

Colorless microcrystals, m.p. $252-253{ }^{\circ} \mathrm{C} .{ }^{1} \mathrm{HNMR}$ $\left(\mathrm{CDCl}_{3}, 500 \mathrm{MHz}\right) \delta=8.16(\mathrm{~s}, 2 \mathrm{H}), 7.77(\mathrm{~s}, 2 \mathrm{H}), 4.17(\mathrm{tt}, J=$ 12.7, $3.4 \mathrm{~Hz}, 1 \mathrm{H}), 2.27$ (qd, $J=12.7,3.4 \mathrm{~Hz}, 2 \mathrm{H}), 1.88(\mathrm{dm}$, $J=12.7,2 \mathrm{H}), 1.71(\mathrm{dm}, J=12.7 \mathrm{~Hz}, 1 \mathrm{H}), 1.39(\mathrm{qd}, J=12.7$, $3.4 \mathrm{~Hz}, 2 \mathrm{H}), 1.30(\mathrm{qd}, J=12.7,3.4 \mathrm{~Hz}, 1 \mathrm{H}) \mathrm{ppm} .{ }^{13} \mathrm{CNMR}$ $\left(126 \mathrm{MHz}, \mathrm{CDCl}_{3}\right) \delta=168.4,139.3,134.3,129.7,127.1$, $123.3,51.0,29.8,26.1,25.2,20.3$ ppm. IR (KBr) $v=1757$ (s), $1701(\mathrm{vs}) \mathrm{cm}^{-1}$. MS $\mathrm{m} / z(\%)=307\left(\mathrm{M}^{+}, 73\right), 264$ (33), 226 (100), 208 (26), 154 (13). HRMS calcd for $\mathrm{C}_{20} \mathrm{H}_{21} \mathrm{NO}_{2}$ $\left(\mathrm{M}^{+}\right)$307.1572, found 307.1570.

\subsection{N-(4-Methoxyphenyl)-6,7-dimethyl-2,3-naphthalimide (17)}

Colorless microcrystals, m.p. $278-279{ }^{\circ} \mathrm{C} .{ }^{1} \mathrm{HNMR}(500$ $\left.\mathrm{MHz}, \mathrm{CDCl}_{3}\right) \delta=8.31$ (s, 2H), $7.82(\mathrm{~s}, 2 \mathrm{H}), 7.39(\mathrm{dm}, J=9.1$ $\mathrm{Hz}, 2 \mathrm{H}), 7.04(\mathrm{dm}, J=9.1 \mathrm{~Hz}, 2 \mathrm{H}), 3.86(\mathrm{~s}, 3 \mathrm{H}), 2.50(\mathrm{~s}, 6 \mathrm{H})$ ppm. ${ }^{13} \mathrm{CNMR}\left(126 \mathrm{MHz}, \mathrm{CDCl}_{3}\right.$ at $\left.50{ }^{\circ} \mathrm{C}\right) \delta=167.5,159.3$, $139.7,134.6,129.9,128.0,127.0,124.9,124.1,114.5,55.6$, 20.3 ppm. IR (KBr) $v=1767(\mathrm{~m}), 1739(\mathrm{~m}), 1714(\mathrm{vs}) \mathrm{cm}^{-1}$. MS $m / z(\%)=331\left(\mathrm{M}^{+}, 100\right), 316(29), 287$ (7), $316(29)$, 208 (7), 154 (7). HRMS calcd for $\mathrm{C}_{21} \mathrm{H}_{17} \mathrm{NO}_{3}\left(\mathrm{M}^{+}\right) 331.1208$, found 331.1207 . 


\subsection{6. $\mathrm{N}$-(4-Bromophenyl)-6,7-dimethyl-2,3-naphthalimide} (18)

Faintly pink solids, m.p. $>300{ }^{\circ} \mathrm{C}$. ${ }^{1} \mathrm{HNMR}(500 \mathrm{MHz}$, $\left.\mathrm{CDCl}_{3}\right) \delta=8.32(\mathrm{~s}, 2 \mathrm{H}), 7.83(\mathrm{~s}, 2 \mathrm{H}), 7.64(\mathrm{dm}, J=8.7 \mathrm{~Hz}$, 2H), $7.40(\mathrm{dm}, J=8.7 \mathrm{~Hz}, 2 \mathrm{H}), 2.51(\mathrm{~s}, 6 \mathrm{H}) \mathrm{ppm} .{ }^{13} \mathrm{CNMR}$ $\left(125 \mathrm{MHz}, \mathrm{CDCl}_{3}\right) \delta=166.9,140.0,134.5,132.2,131.1$, 129.9, 128.0, 127.0, 124.5, 121.7, 20.4 ppm. IR (KBr) $v=$ 1785 (s), 1719 (vs), 1709 (vs) cm cm $^{-1}$ MS m/z (\%) = $381\left(\mathrm{M}^{+}\right.$, 100), 379 ( $\left.\mathrm{M}^{+}, 100\right), 337$ (21), 335 (21), 154 (17), 153 (12), 152 (12), 150 (11), 143 (11), 139 (11). HRMS calcd for $\mathrm{C}_{20} \mathrm{H}_{14}{ }^{79} \mathrm{BrNO}_{2}\left(\mathrm{M}^{+}\right)$379.0208, found 379.0210.

\subsection{6,7-Dichloro-N-phenyl-2,3-naphthalimide (19)}

Yellowish powder, m.p. $>300{ }^{\circ} \mathrm{C}$. ${ }^{1} \mathrm{HNMR}(500 \mathrm{MHz}$, DMSO- $d_{6}$ at $\left.140{ }^{\circ} \mathrm{C}\right) \delta=8.57(\mathrm{~s}, 2 \mathrm{H}), 8.54(\mathrm{~s}, 2 \mathrm{H}), 7.52(\mathrm{t}, J$ $=7.4 \mathrm{~Hz}, 2 \mathrm{H}), 7.48(\mathrm{~d}, J=7.4 \mathrm{~Hz}, 2 \mathrm{H}), 7.43(\mathrm{t}, J=7.4 \mathrm{~Hz}$, 1H) ppm. ${ }^{13} \mathrm{CNMR}\left(125 \mathrm{MHz}, \mathrm{DMSO}-d_{6}\right.$ at $\left.140{ }^{\circ} \mathrm{C}\right) \delta=$ $165.2,133.8,131.7,131.6,130.5,128.1,128.0,127.3,126.3$, $123.2 \mathrm{ppm}$. IR (KBr) $v=1772$ (s), 1746 (s), 1718 (vs), 1702 (vs) $\mathrm{cm}^{-1}$. MS $\mathrm{m} / \mathrm{z}(\%)=345\left(\mathrm{M}^{+}, 12\right), 343\left(\mathrm{M}^{+}, 66\right), 341$ (M+, 100), 299 (27), 298 (40), 227 (19), 196 (20), 194 (30). HRMS calcd for $\mathrm{C}_{18} \mathrm{H}_{9}{ }^{35} \mathrm{Cl}_{2} \mathrm{NO}_{2}\left(\mathrm{M}^{+}\right)$341.0010, found 341.0007 .

\subsection{6,7-Dichloro-N-cyclohexyl-2,3-naphthalimide (20)}

Colorless powder, mp $>300{ }^{\circ} \mathrm{C}$. ${ }^{1} \mathrm{HNMR}(500 \mathrm{MHz}$, $\left.\mathrm{CDCl}_{3}\right) \delta=8.20(\mathrm{~s}, 2 \mathrm{H}), 8.15(\mathrm{~s}, 2 \mathrm{H}), 4.19(\mathrm{tt}, J=12.4,3.4$ $\mathrm{Hz}, 1 \mathrm{H}), 2.26(\mathrm{qd}, J=12.4,3.4 \mathrm{~Hz}, 2 \mathrm{H}), 1.89(\mathrm{dm}, J=12.4$ $\mathrm{Hz}, 2 \mathrm{H}), 1.76(\mathrm{dm}, J=12.4 \mathrm{~Hz}, 2 \mathrm{H}), 1.72(\mathrm{dm}, J=12.4 \mathrm{~Hz}$, $1 \mathrm{H}), 1.40$ (qt, $J=12.4,3.4 \mathrm{~Hz}, 2 \mathrm{H}), 1.30$ (qt, $J=12.4,3.4 \mathrm{~Hz}$, 1H) ppm. ${ }^{13} \mathrm{CNMR}\left(125 \mathrm{MHz}, \mathrm{CDCl}_{3}\right) \delta=167.6,134.4$, $134.0,131.0,129.2,123.2,51.6,29.9,26.2,25.3$ ppm. UV $(\mathrm{MeOH}) \lambda=213(\log \varepsilon=4.39), 245 \mathrm{sh}(4.52), 251 \mathrm{sh}$ (4.63), 261 (4.78), 267 (4.84), 280sh (4.13), 295 (4.04), 319 (3.31), 325 (3.29), 334 (3.19), 344 (3.37), 360 (3.47), 369 (3.15) nm. IR (KBr) $v=1767$ (s), 1703 (vs) $\mathrm{cm}^{-1}$. MS m/z $(\%)=350\left(\mathrm{M}^{+}, 5\right), 349\left(\mathrm{M}^{+}, 28\right), 347\left(\mathrm{M}^{+}, 42\right), 306(16), 304$ (24), 270 (11), 269 (10), 266 (100), 250 (16), 248 (23), 223 (10), 196 (10), 194 (15). HRMS calcd for $\mathrm{C}_{18} \mathrm{H}_{15}{ }^{35} \mathrm{Cl}_{2} \mathrm{NO}_{2}$ $\left(\mathrm{M}^{+}\right)$347.0480, found 347.0480.

\subsection{6,7-Dichloro-N-(4-methoxyphenyl)-2,3-naphthalimide} (21)

Yellow powder, m.p. $>300{ }^{\circ} \mathrm{C} .{ }^{1} \mathrm{HNMR}\left(500 \mathrm{MHz}, \mathrm{CDCl}_{3}\right.$ at $\left.50{ }^{\circ} \mathrm{C}\right) \delta=8.33(\mathrm{~s}, 2 \mathrm{H}), 8.19(\mathrm{~s}, 2 \mathrm{H}), 7.39(\mathrm{~d}, J=8.9 \mathrm{~Hz}$, 2H), $7.03(\mathrm{~d}, J=8.9 \mathrm{~Hz}, 2 \mathrm{H}), 3.86(\mathrm{~s}, 3 \mathrm{H}) \mathrm{ppm} .{ }^{13} \mathrm{CNMR}$ $\left(125 \mathrm{MHz}, \mathrm{CDCl}_{3}\right.$ at $\left.50{ }^{\circ} \mathrm{C}\right) \delta=166.5,159.6,134.5,134.4$, $130.9,128.9,127.8,124.5,123.9,114.6,55.6$ ppm. IR (KBr) $v=1772(\mathrm{~m}), 1725(\mathrm{~s}), 1707(\mathrm{vs}) \mathrm{cm}^{-1}$. MS $\mathrm{m} / z(\%)=375$ $\left(\mathrm{M}^{+}, 12\right), 373\left(\mathrm{M}^{+}, 66\right), 371\left(\mathrm{M}^{+}, 100\right), 358$ (18), 356 (27), 194 (11). HRMS calcd for $\mathrm{C}_{19} \mathrm{H}_{11}{ }^{35} \mathrm{Cl}_{2} \mathrm{NO}_{3}\left(\mathrm{M}^{+}\right)$371.0116, found 371.0118 .

\subsection{N-Phenyl-2,3-anthracenedicarboximide (23) [32]}

Yellow microcrystals, m.p. $>300{ }^{\circ} \mathrm{C}$ (lit [19] 368-370 $\left.{ }^{\circ} \mathrm{C}\right)$.
${ }^{1} \mathrm{HNMR}\left(400 \mathrm{MHz}, \mathrm{DMSO}-d_{6}\right) \delta=9.00(\mathrm{~s}, 2 \mathrm{H}), 8.80(\mathrm{~s}, 2 \mathrm{H})$, $8.23(\mathrm{~m}, 2 \mathrm{H}), 7.72(\mathrm{~m}, 2 \mathrm{H}), 7.52(\mathrm{~m}, 5 \mathrm{H}) \mathrm{ppm}$

\subsection{N-Methyl-2,3-anthracenedicarboximide (24)}

Yellow microcrystals, m.p. $>300{ }^{\circ} \mathrm{C} .{ }^{1} \mathrm{H}$ NMR $(400 \mathrm{MHz}$, $\left.\mathrm{CDCl}_{3}\right) \delta=8.63(\mathrm{~s}, 2 \mathrm{H}), 8.50(\mathrm{~s}, 2 \mathrm{H}), 8.08(\mathrm{~m}, 2 \mathrm{H}), 7.62(\mathrm{~m}$, 2H), $3.76(\mathrm{~s}, 3 \mathrm{H}) \mathrm{ppm} .{ }^{13} \mathrm{CNMR}\left(100 \mathrm{MHz}, \mathrm{CDCl}_{3}\right) \delta=$ $168.0,133.3,131.9,130.1,128.5,127.5,126.8,125.8,24.3$ ppm. IR (KBr) $v=1758(\mathrm{~s}), 1701(\mathrm{vs}) \mathrm{cm}^{-1}$. UV $\left(\mathrm{CH}_{3} \mathrm{CN}\right) \lambda$ $\max _{\max }=211(\log \varepsilon=4.08), 239$ (4.57), 276 (4.54), 287 (4.60), 290 (4.60), 291 (4.60), 293 (460), 307sh (4.40), 335sh (3.42), 322sh (3.55), 381 (3.73), 402 (3.78) nm. MS $m / z(\%)=261$ (M+100), 217 (11), 204 (6), 176 (43), 116 (6), 88 (18). HRMS Calcd for $\mathrm{C}_{17} \mathrm{H}_{11} \mathrm{NO}_{2}\left(\mathrm{M}^{+}\right)$261.0790, found 261.0789

\subsection{2. $\mathrm{N}$-Ethyl-2,3-anthracenedicarboximide (25)}

Yellow microcrystals, m.p. $271-272{ }^{\circ} \mathrm{C} .{ }^{1} \mathrm{H}$ NMR (400 $\mathrm{MHz}, \mathrm{DMSO}-d_{6}$ at $\left.80{ }^{\circ} \mathrm{C}\right) . \delta=8.99(\mathrm{~s}, 2 \mathrm{H}), 8.61(\mathrm{~s}, 2 \mathrm{H})$, $8.17(\mathrm{~m}, 2 \mathrm{H}), 7.67(\mathrm{~m}, 2 \mathrm{H}), 3.71(\mathrm{q}, J=7.2 \mathrm{~Hz}, 2 \mathrm{H}), 1.25(\mathrm{t}$, $J=7.2 \mathrm{~Hz}, 3 \mathrm{H}) \mathrm{ppm}{ }^{13} \mathrm{C}$ NMR $\left(100 \mathrm{MHz}, \mathrm{DMSO}-d_{6}\right.$ at 80 $\left.{ }^{\circ} \mathrm{C}\right) \delta=166.6,132.4,131.3,129.6,128.0,127.2,126.3,125.0$, $32.4,13.0 \mathrm{ppm}$. IR (KBr) $v=1759(\mathrm{~s}), 1700(\mathrm{vs}) \mathrm{cm}^{-1}$. MS $m / z(\%)=275\left(\mathrm{M}^{+}, 100\right), 260(54), 247$ (21), 233 (7), 205 (7), 176 (28), 130 (6), 116 (9), 102 (6), 88 (21). HRMS Calcd for $\mathrm{C}_{18} \mathrm{H}_{13} \mathrm{NO}_{2}\left(\mathrm{M}^{+}\right)$275.0946, found 275.0944.

\subsection{3. $\mathrm{N}$-(4-Bromophenyl)-2,3-anthracenedicarboximide (26) [32]}

Yellow microcrystals, m.p. $>300{ }^{\circ} \mathrm{C} .{ }^{1} \mathrm{H}$ NMR $(400 \mathrm{MHz}$, DMSO $-d_{6}$ at $\left.100{ }^{\circ} \mathrm{C}\right) . \delta=8.96(\mathrm{~s}, 2 \mathrm{H}), 8.74(\mathrm{~s}, 2 \mathrm{H}), 8.20(\mathrm{~m}$, 2H), 7.74 (d, $J=8.5 \mathrm{~Hz}, 2 \mathrm{H}), 7.70(\mathrm{~m}, 2 \mathrm{H}), 7.50$ (d, $J=8.5$ $\mathrm{Hz}, 2 \mathrm{H}) \mathrm{ppm}$ IR (KBr) $v=1786(\mathrm{~m}), 1742$ (m), 1717 (vs), 1708 (vs) $\mathrm{cm}^{-1}$. MS m/z (\%) = $403\left(\mathrm{M}^{+}, 100\right), 401\left(\mathrm{M}^{+}, 100\right)$, 359 (34), 277 (17), 203 (29), 176 (88), 161 (30). HRMS Calcd for $\mathrm{C}_{22} \mathrm{H}_{12}{ }^{79} \mathrm{BrNO}_{2}\left(\mathrm{M}^{+}\right)$401.0051, found 401.0052 .

\subsection{N-(4-Methoxyphenyl)-2,3-anthracenedicarboximide (27)}

Yellow microcrystals, m.p. $>300{ }^{\circ} \mathrm{C} .{ }^{1} \mathrm{H}$ NMR $(400 \mathrm{MHz}$, DMSO- $d_{6}$ at $\left.80{ }^{\circ} \mathrm{C}\right) . \delta=8.95(\mathrm{~s}, 2 \mathrm{H}), 8.72(\mathrm{~s}, 2 \mathrm{H}), 8.20$ (m, 2H), $7.69(\mathrm{~m}, 2 \mathrm{H}), 7.41(\mathrm{dm}, J=8.8 \mathrm{~Hz}, 2 \mathrm{H}), 7.09(\mathrm{dm}, J=$ $8.8 \mathrm{~Hz}, 2 \mathrm{H}), 3.84(\mathrm{~s}, 3 \mathrm{H}) \mathrm{ppm} .{ }^{13} \mathrm{C} \mathrm{NMR}(100 \mathrm{MHz}$, DMSO $-d_{6}$ at $\left.100^{\circ} \mathrm{C}\right) \delta=166.1,159.6,158.7,132.4,131.4$, 129.6, 128.0, 127.9, 127.1, 125.4, 124.6, 113.9, 55.2 ppm. IR (KBr) $v=1762(\mathrm{~m}), 1702(\mathrm{vs}) \mathrm{cm}^{-1}$. UV $\left(\mathrm{CH}_{3} \mathrm{CN}\right) \lambda_{\max }=213$ $(\log \varepsilon=4.25), 239$ (4.66), 247 (4.66), 278sh (4.62), 291sh (4.74), 299 (4.80), 382 (3.80), 404 (3.82) nm. MS $m / z(\%)=$ $353\left(\mathrm{M}^{+}, 100\right), 338$ (18), 309 (19), 266 (5), 230 (6), 203 (9), 176 (28). HRMS Calcd for $\mathrm{C}_{23} \mathrm{H}_{15} \mathrm{NO}_{3}\left(\mathrm{M}^{+}\right) 353.1052$, found 353.1049 .

\subsection{5. $N, N$ '-Tetramethylenebis(2,3-naphthalenedicarbox-imide) (31)}

Colorless solids, m.p. $>300{ }^{\circ} \mathrm{C} .{ }^{1} \mathrm{HNMR}\left(500 \mathrm{MHz}, \mathrm{CDCl}_{3}\right.$ at $\left.50{ }^{\circ} \mathrm{C}\right) \delta=8.30(\mathrm{~s}, 4 \mathrm{H}), 8.02(\mathrm{~m}, 4 \mathrm{H}), 7.67(\mathrm{~m}, 4 \mathrm{H}), 3.82$ 
(m, 4H), $1.83(\mathrm{~m}, 4 \mathrm{H}) \mathrm{ppm} .{ }^{13} \mathrm{CNMR}\left(100 \mathrm{MHz}, \mathrm{CDCl}_{3}\right.$ at 50 $\left.{ }^{\circ} \mathrm{C}\right) \delta=168.0,135.6,130.3,129.1,128.0,124.6,37.8,26.1$ ppm. IR (KBr) $v=1762(\mathrm{vs}), 1717(\mathrm{vs}), 1710(\mathrm{vs}) \mathrm{cm}^{-1}$. UV $\left(\mathrm{CHCl}_{3}\right) \lambda_{\max }=246 \mathrm{sh}(\log \varepsilon=4.73), 258 \mathrm{sh}(4.95), 262$ (5.01), 283 (4.14), 293 (4.16), 304 (4.03), 325 (3.51), 343 (3.70), 359 (3.87) nm. MS $m / z(\%)=448\left(\mathrm{M}^{+}, 60\right), 251$ (14), 239 (11), 238 (65), 225 (11), 224 (18), 211 (36), 210 (100), 183 (11), 180 (10), 155 (17), 154 (11), 127 (28), 126 (24). HRMS calcd for $\mathrm{C}_{28} \mathrm{H}_{20} \mathrm{~N}_{2} \mathrm{O}_{4}\left(\mathrm{M}^{+}\right)$448.1423, found 448.1424.

\subsection{6. $N, N$ '-Hexamethylenebis(2,3-naphthalenedicarbox-imide)} (32)

Colorless solids, m.p. $274-276{ }^{\circ} \mathrm{C} .{ }^{1} \mathrm{HNMR}\left(500 \mathrm{MHz}, \mathrm{CDCl}_{3}\right.$ at $\left.40{ }^{\circ} \mathrm{C}\right) \delta=8.30(\mathrm{~s}, 4 \mathrm{H}), 8.03(\mathrm{~m}, 4 \mathrm{H}), 7.67(\mathrm{~m}, 4 \mathrm{H}), 3.74(\mathrm{t}, J$ $=7.0 \mathrm{~Hz}, 4 \mathrm{H}$ ), 1.74 (quin, $J=7.0 \mathrm{~Hz}, 4 \mathrm{H}$ ), 1.44 (quin, $J=7.0$ $\mathrm{Hz}, 4 \mathrm{H}) \mathrm{ppm} .{ }^{13} \mathrm{CNMR}\left(100 \mathrm{MHz}, \mathrm{CDCl}_{3}\right.$ at $\left.40{ }^{\circ} \mathrm{C}\right) \delta=168.0$, $135.5,130.3,129.0,128.0,124.5,38.2,28.4,26.5$ ppm. IR $(\mathrm{KBr}) v=1760(\mathrm{~s}), 1708(\mathrm{vs}) \mathrm{cm}^{-1}$. UV $\left(\mathrm{CHCl}_{3}\right) \lambda_{\max }=244 \mathrm{sh}$ ( $\log \varepsilon=4.70), 255 \mathrm{sh}$ (4.94), 262 (5.06), 281 (4.15), 293 (4.17), 303sh (4.03), 324 (3.48), 343 (3.71), 359 (3.89) nm. MS m/z $(\%)=476\left(\mathrm{M}^{+}, 41\right), 266(25), 238(24), 224$ (13), 211 (43), 210 (100), 180 (11), 155 (14), 127 (21), 126 (17). HRMS calcd for $\mathrm{C}_{30} \mathrm{H}_{24} \mathrm{~N}_{2} \mathrm{O}_{4}\left(\mathrm{M}^{+}\right)$476.1736, found 476.1738.

\subsection{7 .}

\section{N,N'-(m-Phenylene)bis(6,7-dimethyl-2,3-naphtha-lene dicarboximide) (33) [32]}

Orange solids, m.p. $>300{ }^{\circ} \mathrm{C} .{ }^{1} \mathrm{HNMR}\left(500 \mathrm{MHz}, \mathrm{DMSO}-d_{6}\right.$ at $\left.150{ }^{\circ} \mathrm{C}\right) \delta=8.37(\mathrm{~s}, 4 \mathrm{H}), 7.99(\mathrm{~s}, 4 \mathrm{H}), 7.67(\mathrm{~m}, 2 \mathrm{H}), 7.58$ $(\mathrm{dm}, J=7.6 \mathrm{~Hz}, 4 \mathrm{H}), 2.49(\mathrm{~s}, 12 \mathrm{H}) \mathrm{ppm}$. IR (KBr) $v=1766(\mathrm{~s})$, 1731 (s), 1714 (s) $\mathrm{cm}^{-1}$. MS $m / z(\%)=524\left(\mathrm{M}^{+}, 16\right), 368$ (22), 120 (53), 97 (16), 88 (38), 56 (100). HRMS calcd for $\mathrm{C}_{34} \mathrm{H}_{24} \mathrm{~N}_{2} \mathrm{O}_{4}\left(\mathrm{M}^{\dagger}\right)$ 524.1736, found 524.1738.

\section{Conclusion}

We have demonstrated a novel protocol for synthesis of arenedicarboximides. Our phosphine-assisted annulation resulted in much better yields of the products than those by the phosphite-assisted method reported by Haddadins et al. Also, we could successfully apply the method to the synthesis of some diimides.

\section{Acknowledgements}

We thank Mr K. Ariyasu and N. Kobayashi for preparation of some starting materials. A financial support (for M.O.) from the Faculty of Science in Shinshu University is greatly acknowledged.

\section{References}

[1] Y. Maruoka, "Photoreactions of cyclic imides. Examples of synthetic organic photochemistry", Acc. Chem. Res. 1978, 11, 407-413.
[2] A. Demeter, L. Biczók, T. Bérces, V. Wintgens, P. Valat, and J. Kossanyi, "Laser photolysis studies of transient processes in the photoreduction of naphthalimides by aliphatic amines", $J$. Phys. Chem. 1993, 97, 3217-3224.

[3] K. Maruyama and Y. Kubo, "Photochemistry of phthalimides with olefins. Solvent-incorporated addition vs. cycloaddtion to imide $\mathrm{C}(=\mathrm{O})-\mathrm{N}$ bond accompanying ring enlargement", J. Org. Chem. 1985, 50, 1426-1435.

[4] Y. Kubo, M. Suto, T. Arai, P. H. Mazzocchi, L. Klingler, D. Shook, and C. Somich, "Photochemical reactions of $\mathrm{N}$-methylnaphthalene-2,3-dicarboximide with alkenes", J. Org. Chem. 1986, 51, 4404-4411.

[5] P. Valat, V. Wintgens, J. Kossanyi, L. Biczók, A. Demeter, and T. Bérces, "Influence of geometry on the emitting properties of 2,3-naphthalimides", J. Am. Chem. Soc. 1992, 114, 946-953.

[6] A. Demeter, T. Bérces, L. Biczók, V. Wintgens, P. Valat, and J. Kossanyi, "Comprehensive model of the photophysics of $N$-phenylnaphthalimides: The role of solvent and rotational relaxation", J. Phys. Chem. 1996, 100, 2001-2011.

[7] J. C. Netto-Ferreira, V. Wintgens, L. F. V. Ferreira, A. R. Garcia, L. M. Ilharco, and M. J. Lemos, "Solid complexes between $N$-(para-alkylphenyl)-2,3-naphthalimides and $\beta$-cyclodextrin: Characterization by diffuse reflectance infrared Fourier transform spectroscopy and laser induced luminescence studies", J. Photochem. Photobiol. A: Chem. 2000, 132, 209-217.

[8] P. Valat, V. Wintgens, J. Kossanyi, L. Biczók, A. Demeter, and T. Bérces, "Temperature-dependent behavior of the dual fluorescence of 2-(3-fluorophenyl)-2,3-dihydro-1H-benzo[f]isoindole-1,3-dione", Helv. Chim. Acta 2001, 84, 2813-2832.

[9] P. Nandhikonda and M. D. Heagy, "Dual fluorescent $N$-aryl-2,3-naphthalimides: Applications in ratiometric DNA detection and white organic light-emitting devices", Org. Lett. 2010, 12, 4796-4799

[10] L. Ding, H.-B. Li, T. Lei, H.-Z. Ying, R.-B. Wang, Y. Zhou, Z.-M. Su, and J. Pei, "Alkylene-chain effect on microwire growth and crystal packing of $\pi$-moieties", Chem. Mater. 2012, 24, 1944-1949.

[11] Y. Zhong, B. Kumar, S. Oh, M. T. Trinh, Y. Wu, K. Elbert, P. Li, X. Zhu, S. Xiao, F. Ng, M. L. Steigerwald, and C. Nuckolls, "Helical ribbons for molecular electronics", J. Am. Chem. Soc. 2014, 136, 8122-8130.

[12] X. Zhan, A. Facchetti, S. Barlow, T. J. Marks, M. A. Ratner, M. R. Wasielewski, and S. R. Marder, "Rylene and related diimides for organic electronics", Adv. Mater. 2011, 23, 268-284.

[13] S. Guha, F. S. Goodson, L. J. Corson, and S. Saha, "Boundaries of anion/naphthalenediimide interactions; From anion- $\pi$ interactions to anion-induced charge-transfer and electron-transfer phenomena", J. Am. Chem. Soc. 2012, 134, 13679-13691.

[14] H. Cao, V. Chang, R. Hernandez, and M. D. Heagy, "Matrix screening of substituted $\mathrm{N}$-aryl-1,8-naphthalimides reveals new dual fluorescent dyes and unusually bright pyridine derivatives", J. Org. Chem. 2005, 70, 4929-4934.

[15] K. Imaizumi, H. Terasima, K. Akasaka, and H. Ohrui, "Highly potent chiral labeling reagents for the discrimination of chiral alcohols", Anal. Sci. 2003, 19, 1243-1249. 
[16] L. Kürti and B. Czakó, Strategic applications of named reactions in organic synthesis, pp.182-183, Elsevier Academic Press, Amsterdat, 2005.

[17] M. Sato, S. Ebine, and S. Akabori, "Condensation of halobenzenes and haloferrocenes with phthalimide in the presence of copper(I) oxide; A simplified Gabriel reaction", Synthesis 1981, 472-473.

[18] S. Kuroda, N. Tamura, R. Miyatake, N. Matsumoto, Y. Horino, D. Miyawaki, and M. Oda, "Synthesis and emission properties of 1,6-methano[10]annulene-3,4-dicarboximides, Heterocycles 2011, 83, 789-796.

[19] M. P. Cava amd P. L. Shirley, "Condensed cyclobutane aromatic compounds. X. Naphtho[b]cyclobutene", J. Am. Chem. Soc. 1960, 82, 654-656.

[20] T. Iwanaga, R. Tanaka, and S. Toyota, "Introduction of an arylethynyl group onto an anthracene bisimide core for molecular design of new $\pi$-conjugated compounds", Chem. Lett. 2014, 43, 105-107

[21] Y. Nishina, T. Kida, and T. Ureshino, "Facile $\mathrm{Sc}(\mathrm{OTf})_{3}$-catalyzed generation and successive aromatization of isobenzofuran from o-dicarbonylbenzenes", Org. Lett. 2011, 13, 3960-3963.

[22] C. R. Hickenboth, J. S. Moore, S. R. White, N. R. Sottos, J. Baudry, and S. R Wilson, "Biasing reaction pathways with mechanical force", Nature 2007, 446, 423-427.

[23] M. J. Haddadin, B. J. Agha, and R. F. Tabri, "Syntheses of some furans and naphtho[2,3-c] derivatives of furan, pyrrole, and thiophene", J. Org. Chem. 1979, 44, 494-497.

[24] O. Farooq, "Oxidation of aromatic 1,2-dimethanols by activated dimethyl sulfoxide", Synthesis 1994, 1035-1036.
[25] Y. Cheng, J.-H. Peng, Y.-J. Li, X.-Y. Shi, M.-S. Tang, and T.-Y Tan, " $N$-Heterocyclic carbene catalyzed reaction of phthalaldehydes: Controllable stereoselective synthesis of polyhydroxylated spiro- and fused indenones dictated by the structure of NHC catalysts", J. Org. Chem. 2011, 76, 1844-1851.

[26] R. Tona and R. Häner, "Crosslinking of diene-modified DNA with bis-maleimides", Mol. BioSyst. 2005, 1, 93-98.

[27] L. Kürti and B. Czakó, Strategic applications of named reactions in organic synthesis, pp.48-49, Elsevier Academic Press, Amsterdat, 2005; V. Nair and K. G. Abhilash, "Engaging Morita-Baylis-Hillman reaction for the generation of isobenzofuran and the consequent entry into highly substituted aromatic systems", Synthesis 2005, 1967-1970

[28] E. Hedaya and S. Theodoropulos, "The preparations and ractions of stable phosphorus ylides derived from maleic anhydrides, maleimides or isomaleimides", Tetrahedron 1968, 24 , 2241-2254. Tri- $n$-octylylphosphorane, prepared by reaction of tri- $n$-octylphosphine and $N$-phenylmaleimide in refluxing dioxane in situ, was used.

[29] M. P. Cava, A. A. Deana, K. Muth, and M. J. Mitchell, "N-Phenylmaleimide", Org. Synth. Coll. Vol. 5, 1973, 944-946.

[30] A Mallouli and Y. Lepage, "Convenient syntheses of naphthalene-, anthracene-, and naphthacene-2,3-dicarbaldehydes”, Synthesis 1980, 689.

[31] J. Rigaudy and D. Sparfel, "Thermal tansformations of meso-acene photooxides. V. Influence of meso-phenyl substituents in naphthacenic series", Tetrahedron 1978, 34, 2263-2273.

[32] Because of its low solubility, its satisfactory ${ }^{13} \mathrm{C}$ NMR spectrum was not recorded. 